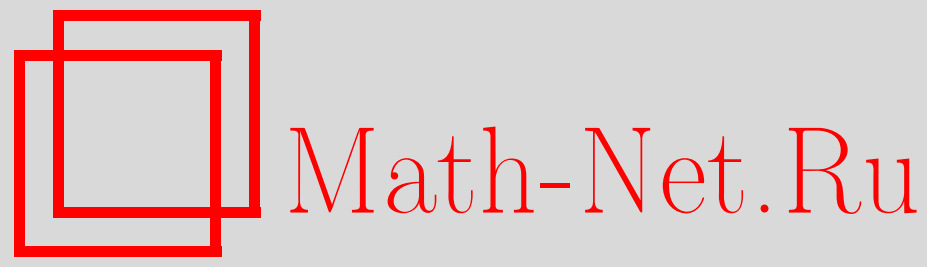

И. Качковский, Отсутствие собственных значений у периодического оператора Шрёдингера с сингулярным потенциалом в прямоугольном цилиндре, Функи. анализ и его прил., 2013, том 47, выпуск $2,27-37$

DOI: https://doi.org/10.4213/faa3107

Использование Общероссийского математического портала Math$\mathrm{Net.Ru} \mathrm{подразумевает,} \mathrm{что} \mathrm{вы} \mathrm{прочитали} \mathrm{и} \mathrm{согласны} \mathrm{с} \mathrm{пользователь-}$ ским соглашением

http://www . mathnet.ru/rus/agreement

Параметры загрузки:

IP : 35.173 .219 .12

26 апреля 2023 г., 12:31:02

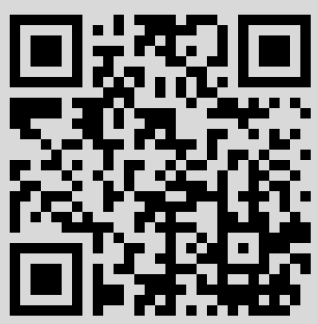




\title{
Отсутствие собственных значений у периодического оператора Шрёдингера с сингулярным потенциалом в прямоугольном цилиндре*
}

\author{
(с) 2013. И. В. КАчковСКиЙ
}

В работе рассматривается периодический оператор Шрёдингера в $d$-мерном цилиндре с прямоугольным сечением, $d \geqslant 3$. Электрический потенциал может иметь сингулярную часть вида $\sigma(x, y) \delta_{\Sigma}(x, y)$, где $\Sigma-$ периодическая система гиперповерхностей. Устанавливается, что в спектре такого оператора отсутствуют собственные значения, если $\Sigma$ обладает достаточной гладкостью и $\sigma \in L_{p, \operatorname{loc}}(\Sigma), p>d-1$.

\section{§1. Введение}

Пусть $\Gamma \subset \mathbb{R}^{m}$ - решетка,

$$
\Gamma=\left\{l_{1} b_{1}+\cdots+l_{m} b_{m}, l_{j} \in \mathbb{Z}\right\},
$$

где $b_{1}, \ldots, b_{m}-$ фиксированный (не обязательно ортонормированный) базис в $\mathbb{R}^{m}$. Будем считать, что $\left|b_{1}\right|=1$; условия теорем будут инвариантны относительно масштабирований по соответствующей координате. Пусть

$$
\Omega=\left\{y_{1} b_{1}+\cdots+y_{m} b_{m}, 0 \leqslant y_{i}<1\right\}
$$

- элементарная ячейка решетки Г. Множество $\Omega$ естественно отождествляется с множеством точек $m$-мерного тора $\mathbb{T}^{m}=\mathbb{R}^{m} / \Gamma$. Пусть $U=\left[0 ; a_{1}\right] \times \cdots \times\left[0 ; a_{k}\right]$ $\subset \mathbb{R}^{k}, a_{i}>0$, - параллелепипед в $\mathbb{R}^{k}, k \geqslant 0$. Пусть $d=k+m$. Через $\operatorname{mes}_{d-1}$ будем обозначать $(d-1)$-мерную меру Лебега.

Определение 1. $(d-1)$-мерное липшицево подмногообразие $\Sigma_{0} \subset U \times \mathbb{T}^{m}$ будем называть $C^{l}$-гиперповерхностью, если существует $C^{l}$-гладкое компактное подмногообразие

$$
\widetilde{\Sigma}_{0} \subset \mathbb{R}^{k} \times \mathbb{T}^{m}
$$

без края или с $C^{l}$-гладким краем, такое, что $\Sigma_{0}=\widetilde{\Sigma}_{0} \cap\left(U \times \mathbb{T}^{m}\right)$ и $\operatorname{mes}_{d-1}\left(\Sigma_{0}\right)>0$.

Определение 2. Г-периодической системой $C^{l}$-гиперповерхностей в цилиндре $\Xi=U \times \mathbb{R}^{m}$ будем называть подмножество вида

$$
\Sigma=\bigcup_{j=1}^{j_{0}} \bigcup_{n \in \Gamma}\left(\Sigma_{j}+n\right) \subset \Xi,
$$

где вектор $n \in \Gamma \subset \mathbb{R}^{m}$ отождествляется с вектором $(0, n) \in \mathbb{R}^{d}$, а $\Sigma_{j} \subset U \times \Omega$, рассматриваемые как подмногообразия в $U \times \mathbb{T}^{m}$, являются $C^{l}$-гладкими гиперповерхностями.

*Работа выполнена при поддержке King's Annual Fund Studentship и King's Overseas Research Studentship, King's College, London. 
Мы будем рассматривать матричный периодический оператор Шрёдингера

$$
H=-\Delta+V(x, y)+\sigma(x, y) \delta_{\Sigma}(x, y),
$$

в пространстве $L_{2}\left(\Xi ; \mathbb{C}^{N}\right)$, где $\Xi=U \times \mathbb{R}^{m} \subset \mathbb{R}^{d}, d=k+m \geqslant 3$. Подразумевается, что при $k=0$ цилиндр $\Xi-$ это все пространство $\mathbb{R}^{d}$, а при $k=1$ это плоско-параллельный слой $\Xi=[0, a] \times \mathbb{R}^{d-1}$. Нам понадобится общий вариант краевых условий: на каждой паре противоположных граней цилиндра $\Xi$ для каждой из $N$ компонент вектора $u$ мы задаем условие Дирихле или Неймана (при $k=0$ краевые условия отсутствуют). Мы предполагаем, что $\Sigma$ есть $Г$-периодическая система $C^{d}$-гиперповерхностей в $\Xi$. Через $M_{N}(\mathbb{C})$ будем обозначать пространство $N \times N$-матриц с комплексными элементами. Матричнозначные функции

$$
\sigma \in L_{d-1, \text { loc }}\left(\Sigma ; M_{N}(\mathbb{C})\right) \quad \text { и } \quad V \in L_{d / 2, \text { loc }}\left(U \times \mathbb{R}^{m} ; M_{N}(\mathbb{C})\right)
$$

предполагаются периодическими по «продольным» переменным,

$$
\sigma(x, y+a)=\sigma(x, y), \quad V(x, y+a)=V(x, y), \quad(x, y) \in \Xi, a \in \Gamma .
$$

Мы не исключаем ситуации, в которой $\Sigma \cap \partial \Xi \neq \varnothing$. Таким образом, результат охватывает случай краевого условия третьего типа

$$
\frac{\partial u}{\partial n}(x, y)+\sigma(x, y) u(x, y)=0, \quad(x, y) \in \partial \Xi .
$$

Чтобы дать точное определение оператора $H$, рассмотрим квадратичную форму

$$
\begin{aligned}
h[u]=\int_{\Xi}|\nabla u(x, y)|^{2} d x d y+\int_{\Xi}\langle V(x, y) & u(x, y), u(x, y)\rangle d x d y \\
& +\int_{\Sigma}\langle\sigma(x, y) u(x, y), u(x, y)\rangle d S(x, y),
\end{aligned}
$$

где $d S$ - поверхностная мера на $\Sigma$, а $\langle\cdot, \cdot\rangle$ - стандартное скалярное произведение в $\mathbb{C}^{N}$. Квадратичная форма $h$ определена на области

$$
H_{\mathscr{C}}^{1}\left(\Xi ; \mathbb{C}^{N}\right)=\left\{u \in H^{1}\left(\Xi ; \mathbb{C}^{N}\right):\left.u_{i}(x, y)\right|_{x_{j}=0}=\left.u_{i}(x, y)\right|_{x_{j}=a_{j}}=0 \text { при }(i, j) \in \mathscr{C}\right\},
$$

где $\mathscr{C} \subset\{1, \ldots, N\} \times\{1, \ldots, k\}-$ некоторый фиксированный набор пар индексов, определяющий, на каких гранях и для каких компонент функции заданы условия Дирихле. Нетрудно показать, что эта форма замкнута и секториальна в смысле Като [5] и отвечает некоторому $m$-секториальному оператору $H$, который мы будем называть матричным периодическим оператором Шрёдингера в прямоугольном цилиндре со смешанными краевыми условиями, см. также [15]. При эрмитовых $V$ и $\sigma$ оператор $H$ самосопряжен и полуограничен снизу.

Теорема 3. Пусть $d=k+m \geqslant 3, m \geqslant 1$. Пусть $\Sigma \subset \Xi=U \times \mathbb{R}^{m}$ eсmъ $\Gamma$-периодическая система $C^{d}$-гиперповерхностей. Пусть $\sigma \in L_{p, \operatorname{loc}}\left(\Sigma ; M_{N}(\mathbb{C})\right)$, $p>d-1, u V \in L_{d / 2, \operatorname{loc}}\left(\Xi ; M_{N}(\mathbb{C})\right)$ - некоторые $\Gamma$-периодические функиии. Тогда у соответствующего оператора $\mathrm{H}$ в $L_{2}(\Xi)$ нет собственных значений. Если $H=H^{*}$, то его спектр абсолютно непрерывен. 
Доказательство отсутствия собственных значений в несамосопряженном матричном случае проводится точно так же, как и доказательство абсолютной непрерывности спектра в скалярном случае с вещественными $V$ и $\sigma$. Однако в приложениях теорема 3 может понадобиться в общей формулировке. В частности, вопрос об абсолютной непрерывности спектра самосопряженного оператора Максвелла в слое $[0 ; a] \times \mathbb{R}^{2}$ (см. [16]) или в параллелепипеде $\left[0 ; a_{1}\right] \times\left[0 ; a_{2}\right] \times \mathbb{R}$ (см. [11]) сводится к отсутствию собственных значений у оператора Шрёдингера с несамосопряженными $V$ и $\sigma, N=6$ (при этом три компоненты удовлетворяют условию Дирихле, а три другие - условию Неймана).

Мы не рассматриваем двумерный случай, так как он изучен в большой общности (см. [1], [13], [18], [14], [21]). В частности, в [21] рассмотрен оператор $H_{d=2}$, отвечающий квадратичной форме

$$
h_{d=2}[u]=\int_{\Xi}|\nabla u(x, y)|^{2} d x d y+\int_{\Xi}|u(x, y)|^{2} d \nu(x, y) .
$$

На периодический заряд $d \nu$ накладывается условие подчиненности. Ему, например, удовлетворяют заряды $d \nu$, такие, что для любого положительного $\varepsilon$ выполняется оценка

$$
\int_{U \times \Omega}|u|^{2}|d \nu| \leqslant \varepsilon \int_{U \times \Omega}|\nabla u|^{2} d x d y+C(\varepsilon) \int_{U \times \Omega}|u|^{2} d x d y \quad \forall u \in H^{1}(U \times \Omega) .
$$

При таком условии спектр оператора $H_{d=2}$ абсолютно непрерывен. Этот результат включает в себя

$$
V \in L_{p, \mathrm{loc}}(\Xi) \quad \text { и } \quad \sigma \in L_{p, \mathrm{loc}}(\Sigma), \quad p>1
$$

(оптимальные условия на $V$ и $\sigma$ в шкале $L_{p}$ при $d=2$ ). Результат в [21] получен и для всей плоскости, и для периодического волновода на плоскости (и, более того, для оператора с магнитным потенциалом). В многомерном случае получить результат в такой общности не удается.

Вопрос об отсутствии собственных значений периодического оператора Шрёдингера исследуется, начиная с пионерской работы Томаса [19], где была установлена абсолютная непрерывность спектра оператора

$$
H_{V}=-\Delta+V
$$

в пространстве $\mathbb{R}^{3}$ с вещественным периодическим потенциалом $V \in L_{2}(\Omega)$. В дальнейшем спектр оператора $H_{V}$ в случае $\mathbb{R}^{d}$ интенсивно изучался, см. например, обзор [2]. Оптимальный в шкале $L_{p}$ результат был установлен в [20]: спектр $H_{V}$ абсолютно непрерывен при $V \in L_{d / 2}(\Omega), d \geqslant 3$.

Оператор Шрёдингера

$$
H_{\sigma}=-\Delta+\sigma \delta_{\Sigma}
$$

в $\mathbb{R}^{d}$ с сингулярным электрическим потенциалом рассматривался, насколько известно автору, в двух работах. В [17] абсолютная непрерывность спектра оператора $H_{\sigma}$ установлена при $d=3$, кусочно $C^{3}$-гладкой поверхности $\Sigma$ и $\sigma \in L_{2, \text { lос }}(\Sigma)$. В этой работе рассматривались и более высокие размерности, однако на (кусочно $C^{2}$-гладкую) поверхность $\Sigma$ накладывалось дополнительное геометрическое условие - существование направления, трансверсального к $\Sigma$ во всех ее точках (такому условию удовлетворяют, например, многогранники, но не удовлетворяет сфера). В предыдущей работе автора [6] рассмотрен случай $d=4$, поверхность $\Sigma \in C^{4}$ подчинена другому геометрическому условию 
- ее гауссова кривизна нигде не обращается в нуль (здесь, наоборот, подходит сфера, но не подходит многогранник; поверхность цилиндра не удовлетворяет ни условиям из [17], ни условиям из [6]). Преимуществом теоремы 3 является отсутствие каких бы то ни было геометрических условий на $\Sigma$.

Периодический оператор в многомерном цилиндре изучен сравнительно мало. В работе [15] установлена абсолютная непрерывность спектра оператора Шрёдингера в слое $(k=1)$ с третьим краевым условием $(4)$ при

$$
\sigma \in L_{q, \text { lос }}(\partial \Xi), \quad q=2 \text { при } d=3, q=2 d-2 \text { при } d \geqslant 4 .
$$

Этот результат легко переносится на случай прямоугольного цилиндра с теми же условиями на $q$ (при $d>3$ условие $q>d-1$ лучше). В цилиндре $\Xi$ с сечением общего вида возникают трудности уже с обычным электрическим потенциалом (при условиях Дирихле или Неймана, см. [7], [8]). Вопрос об абсолютной непрерывности спектра оператора Шрёдингера в цилиндре с третьим краевым условием остается открытым (даже в случае $V=0, \sigma \in C^{\infty}(\partial \Xi)$, если $\sigma$ нетривиально зависит от «продольных» переменных). Отметим, что методы из [17] и [6] в случае $\Sigma=\partial \Xi$ не работают (заведомо нет трансверсальных направлений и гауссова кривизна равна нулю).

Мы используем метод работы [8], опирающийся на оценки спектральных проекторов оператора Лапласа. Соответствующие оценки сужений спектральных проекторов на подмногообразия заимствованы из [3]. Выигрыш такого метода также в том, что мы единым образом охватываем сингулярный электрический потенциал и третье краевое условие.

В §2 формулируется критерий Томаса отсутствия собственных значений. Теорема 3 сводится к оценке Томаса (теореме 4). В 33 строится вспомогательный оператор с периодическими краевыми условиями, для которого, с использованием результатов из [3] и [4], доказываются необходимые оценки. В §4 эти оценки распространяются на исходный оператор. Теорема 4 доказывается в §5. Прием с отражениями, примененный в 4 , впервые был использован в доказательстве абсолютной непрерывности в работе [13], см. также [14], [18].

Автор благодарит своего научного руководителя Н. Д. Филонова за постановку задачи и постоянное внимание к работе, а также Т. А. Суслину за ценные замечания.

\section{§2. Схема Томаса}

Для $\xi \in \mathbb{C}^{m}$ рассмотрим операторы $H(\xi)$ в пространстве $L_{2}\left(U \times \Omega ; \mathbb{C}^{N}\right)$, задаваемые семейством квадратичных форм

$$
\begin{aligned}
h(\xi)[v, v]= & \int_{U \times \Omega}\left\langle\left(\nabla_{y}+i \xi\right) v(x, y),\left(\nabla_{y}+i \bar{\xi}\right) v(x, y)\right\rangle d x d y \\
& +\int_{U \times \Omega}\left\langle\nabla_{x} v(x, y), \nabla_{x} v(x, y)\right\rangle d x d y+\int_{U \times \Omega}\langle V(x, y) v(x, y), v(x, y)\rangle d x d y \\
& +\int_{\Sigma \cap(U \times \Omega)}\langle\sigma(x, y) v(x, y), v(x, y)\rangle d S(x, y) .
\end{aligned}
$$

Областью определения этих форм является пространство Соболева $H_{\mathscr{C}, \mathrm{per}}^{1}(U \times$ $\left.\Omega ; \mathbb{C}^{N}\right)$ с условиями $(6)$ на $\partial U \times \Omega$ и периодическими краевыми условиями на 
$U \times \partial \Omega$. Операторы $H(\xi)$ являются секториальными в смысле Като и аналитически зависят от $\xi$.

Вложение $H_{\mathscr{C}, \text { per }}^{1}\left(U \times \Omega ; \mathbb{C}^{N}\right) \subset L_{2}\left(U \times \Omega ; \mathbb{C}^{N}\right)$ компактно, поэтому спектры операторов $H(\xi)$ дискретны. Согласно критерию Томаса (см. [19], [12], [2], а также [9], [10] для случая несамосопряженного оператора $H$ ), достаточно показать, что у $H(\xi)$ нет постоянных (по $\xi \in \mathbb{C}^{m}$ ) собственных значений. Таким образом, утверждение теоремы 3 вытекает из следующей теоремы.

Теорема 4. Пусть выполнены условия теоремы 3. Для любых $\lambda \in \mathbb{C} u$ $\xi \in \mathbb{R}^{m}, \xi \perp b_{1}$, существует такое $\tau_{0}$, что при $\tau>\tau_{0}$ оператор $(H((\pi+$ $\left.\left.i \tau) b_{1}+\xi\right)-\lambda I\right)$ обратим и

$$
\left\|\left(H\left((\pi+i \tau) b_{1}+\xi\right)-\lambda I\right)^{-1}\right\| \leqslant C \tau^{-1} .
$$

\section{§3. Оператор с периодическими краевыми условиями}

Пусть $\widetilde{U}=\left(-a_{1} ; a_{1}\right] \times \cdots \times\left(-a_{k} ; a_{k}\right]$. В $L_{2}(\widetilde{U} \times \Omega)$ введем вспомогательный оператор $\widehat{H}_{0}(\tau)$, заданный квадратичной формой

$$
\begin{array}{r}
\hat{h}(\tau)[v, v]=\int_{\widetilde{U} \times \Omega}\left\langle\left(\nabla_{y}+i\left((\pi+i \tau) b_{1}+\xi\right)\right) v(x, y),\left(\nabla_{y}+i\left((\pi-i \tau) b_{1}+\xi\right)\right) v(x, y)\right\rangle d x d y \\
+\int_{\widetilde{U} \times \Omega}\left\langle\nabla_{x} v(x, y), \nabla_{x} v(x, y)\right\rangle d x d y
\end{array}
$$

на области определения $H_{\mathrm{per}}^{1}(\widetilde{U} \times \Omega)$ (с периодическими краевыми условиями по всем переменным). Его собственными значениями будут

$$
\hat{h}_{\hat{n} n}(\tau)=\left|n+\pi b_{1}+\xi\right|^{2}-\tau^{2}+|\hat{n}|^{2}+2 i \tau\left\langle n+\pi b_{1}, b_{1}\right\rangle, \quad \hat{n} \in \widetilde{\Gamma}^{\prime}, n \in \Gamma^{\prime},
$$

а собственные функции имеют вид $|\Omega|^{-1 / 2}|\widetilde{U}|^{-1 / 2} e^{i(\hat{n} x+n y)}$, где

$$
\Gamma^{\prime}=\left\{\sum_{i=1}^{m} n_{i} b_{i}^{\prime}\right\}, \quad n_{i} \in \mathbb{Z},\left\langle b_{i}^{\prime}, b_{j}\right\rangle=2 \pi \delta_{i j}
$$

- двойственная к Г решетка, а

$$
\widetilde{\Gamma}^{\prime}=\left\{\hat{n}=\left(\pi m_{1} / a_{1}, \ldots, \pi m_{k} / a_{k}\right), m_{i} \in \mathbb{Z}\right\}
$$

- решетка, двойственная к решетке $\widetilde{\Gamma} \subset \mathbb{R}^{k}$ с элементарной ячейкой $\widetilde{U}$. При $\tau>0$ оператор $\widehat{H}_{0}(\tau)$ обратим и $\left\|\widehat{H}_{0}(\tau)^{-1}\right\| \leqslant(2 \pi \tau)^{-1}$, поскольку

$$
\left|\hat{h}_{\hat{n} n}(\tau)\right| \geqslant 2 \tau\left|\left\langle n+\pi b_{1}, b_{1}\right\rangle\right|=2 \pi \tau\left|2 n_{1}+1\right| \geqslant 2 \pi \tau,
$$

так как $n_{1} \in \mathbb{Z}$.

Из определения $\Sigma$ следует, что $\Sigma \cap(U \times \Omega)=\widetilde{\Sigma} \cap(U \times \Omega)$ для некоторого $\widetilde{\Sigma} \subset(\widetilde{U} \times \Omega)$, где $\widetilde{\Sigma}$, рассматриваемое как подмножество тора $\left(\mathbb{R}^{k} / \widetilde{\Gamma}\right) \times\left(\mathbb{R}^{m} / \Gamma\right)$, является конечным объединением $C^{l}$-гладких $(d-1)$-мерных подмногообразий.

Нам понадобится следующий результат из [3].

Предложение 5. Пусть $M$ есть $C^{\infty}$-гладкое компактное $d$-мерное риманово многообразие без края, $d \geqslant 3, u S \subset M-$ его $C^{d}$-подмногообразие размерности $d-1$. Пусть $-\Delta$ - оператор Лапласа-Бельтрами на $M$ и $E_{\mu}=$ 
$E_{-\Delta}\left[(\mu-1)^{2} ; \mu^{2}\right), \mu \in \mathbb{N},-$ его спектральный проектор в $L_{2}(M)$. Тогда для $u \in L_{2}(M)$

$$
\left\|E_{\mu} u\right\|_{L_{q}(S)} \leqslant C \mu^{\frac{d-1}{2}-\frac{d-1}{q}}\|u\|_{L_{2}(M)}, \quad \frac{2 d}{d-1}<q \leqslant+\infty .
$$

Отметим, что левая часть определена корректно, так как $E_{\mu} u \in H^{s}(M)$ для любого $s>0$ и, следовательно, допускает $L_{q}$-следы на подмногообразиях. Ясно также, что оценка (11) сохраняет силу, если $S$ - конечное объединение $C^{d}$-гиперповерхностей.

Замечание 6. В [3] предложение 5 доказано для $S \in C^{\infty}$, однако можно проследить, что доказательство сохраняет силу для $S \in C^{d}$.

Лемма 7. Пусть $0<\delta<1 / 2, b \geqslant 1$, и пусть $\left|m_{\mu}\right| \leqslant b$ для любого $\mu \in \mathbb{N}$. Тогда

$$
\sum_{\mu=1}^{\infty} \frac{\mu^{1-2 \delta}}{\left|\left(\mu+m_{\mu}\right)^{2}-\tau^{2}\right|+\tau} \leqslant C(b, \delta) \tau^{-\delta}
$$

при $\tau>1$.

Доказательство. Ясно, что оценка верна для любого фиксированного конечного числа слагаемых. Поэтому достаточно оценивать сумму только по $\mu \geqslant 2 b$. Заметим, что

$$
\sum_{\mu \in[\tau-b-1 ; \tau+b]} \frac{\mu^{1-2 \delta}}{\left|\left(\mu+m_{\mu}\right)^{2}-\tau^{2}\right|+\tau} \leqslant(2 b+1) \frac{(\tau+b)^{1-2 \delta}}{\tau} \leqslant C(b) \tau^{-2 \delta}, \quad \tau>1,
$$

так что далее можно ограничиться $\mu \notin[\tau-b-1 ; \tau+b]$. В этом случае можно заменить $m_{\mu}$ на целые числа (возможно, увеличив $b$ на 1 ) так, чтобы все слагаемые разве лишь увеличились. Таким образом, достаточно предполагать, что $m_{\mu} \in \mathbb{Z} \cap[-b ; b]$. Последнее множество конечно и не зависит от $\mu$. Разбив сумму на несколько, можно свести оценку к случаю $m_{\mu}=$ const. Наконец, в сумме с фиксированным $m_{\mu}$ заменим переменную суммирования на $\mu+m_{\mu}$. С точностью до множителя, зависящего только от $b$, оценка такой суммы сведется к оценке величины

$$
\sum_{\mu=1}^{\infty} \frac{\mu^{1-2 \delta}}{\left|\mu^{2}-\tau^{2}\right|+\tau}
$$

Заметим, что

$$
\sum_{\mu^{2} \geqslant 2 \tau^{2}} \frac{\mu^{1-2 \delta}}{\mu^{2}-\tau^{2}+\tau} \leqslant 2 \sum_{\mu^{2} \geqslant 2 \tau^{2}} \mu^{-1-2 \delta} \leqslant C(\delta) \tau^{-2 \delta} .
$$

Далее,

$$
\sum_{\mu<2 \tau} \frac{\mu^{1-2 \delta}}{\left|\mu^{2}-\tau^{2}\right|+\tau} \leqslant 2 \tau^{1-2 \delta} \sum_{\mu<2 \tau} \frac{1}{\left|\mu^{2}-\tau^{2}\right|+\tau} \leqslant 2 \tau^{-2 \delta} \sum_{\mu<2 \tau} \frac{1}{|\mu-\tau|+1} .
$$

Ограниченность последней суммы следует из ограниченности интеграла

$$
\tau^{-2 \delta} \int_{0}^{2 \tau} \frac{d \mu}{|\mu-\tau|+1}=2 \tau^{-2 \delta} \int_{\tau}^{2 \tau} \frac{d \mu}{\mu-\tau+1}=2 \tau^{-2 \delta} \ln (\tau+1) \leqslant C(\delta) \tau^{-\delta} .
$$


При $\tau \neq 0$ введем оператор $\left|\widehat{H}_{0}(\tau)\right|^{-1 / 2}$ с теми же собственными функциями, что и у $\widehat{H}_{0}(\tau)$, и собственными числами $\left|\hat{h}_{\hat{n} n}(\tau)\right|^{-1 / 2}$.

Лемма 8. При $\tau>1 u u \in L_{2}(\widetilde{U} \times \Omega)$

$$
\left\|\left|\widehat{H}_{0}(\tau)\right|^{-1 / 2} u\right\|_{L_{q}(\widetilde{\Sigma})}^{2} \leqslant C(q, \tau)\|u\|_{L_{2}(\widetilde{U} \times \Omega)}^{2}, \quad 1 \leqslant q<\frac{2(d-1)}{d-2},
$$

где $C(q, \tau) \rightarrow 0$ при $\tau \rightarrow+\infty$.

Доказательство. Заметим, что при фиксированном $\tau$ выполняется оценка $\left|\hat{h}_{\hat{n} n}(\tau)\right| \geqslant C\left(|\hat{n}|^{2}+|n|^{2}\right)$, откуда следует, что $\left|\widehat{H}_{0}(\tau)\right|^{-1 / 2} u \in H^{1}(\widetilde{U} \times \Omega)$, и $L_{q}$-след в левой части определен корректно.

Пусть $E_{\mu}-$ спектральный проектор оператора $-\Delta$ на многообразии $\widetilde{U} \times \Omega$ (которое в силу периодических краевых условий отождествляется с $d$-мерным тором) на отрезок $\left[(\mu-1)^{2} ; \mu^{2}\right)$. Поскольку $\operatorname{mes}_{d-1}(\widetilde{\Sigma})<+\infty$, из утверждения леммы для $q_{0}$ следует утверждение для $1 \leqslant q \leqslant q_{0}$. Поэтому можно ограничиться случаем $2 d /(d-1)<q<2(d-1) /(d-2)$ и применить неравенство (11). Заметим, что при рассматриваемых $q$ оно выполняется с показателем $(d-1) / 2-(d-1) / q=1 / 2-\delta$ для некоторого $0<\delta<1 / 2$. Следовательно,

$$
\begin{aligned}
\left\|\left|\widehat{H}_{0}(\tau)\right|^{-1 / 2} u\right\|_{L_{q}(\widetilde{\Sigma})} & \leqslant \sum_{\mu=1}^{\infty}\left\|E_{\mu}\left|\widehat{H}_{0}(\tau)\right|^{-1 / 2} u\right\|_{L_{q}(\widetilde{\Sigma})} \\
& \leqslant C \sum_{\mu=1}^{\infty} \mu^{1 / 2-\delta}\left\|E_{\mu}\left|\widehat{H}_{0}(\tau)\right|^{-1 / 2} u\right\|_{L_{2}(\widetilde{U} \times \Omega)} \\
& \leqslant C \sum_{\mu=1}^{\infty} \mu^{1 / 2-\delta}\left\|E_{\mu}\left|\widehat{H}_{0}(\tau)\right|^{-1 / 2}\right\| \cdot\left\|E_{\mu} u\right\|_{L_{2}(\widetilde{U} \times \Omega)},
\end{aligned}
$$

где в последнем неравенстве мы воспользовались тем, что $\left|\widehat{H}_{0}(\tau)\right|^{-1 / 2}$ коммутирует с $-\Delta$. Далее, по неравенству Коши-Буняковского-Шварца

$$
\left\|\left|\widehat{H}_{0}(\tau)\right|^{-1 / 2} u\right\|_{L_{q}(\widetilde{\Sigma})}^{2} \leqslant C\|u\|_{L_{2}(\widetilde{U} \times \Omega)}^{2}\left(\sum_{\mu=1}^{[b]+1}+\sum_{\mu=[b]+2}^{+\infty}\right) \mu^{1-2 \delta}\left\|E_{\mu}\left|\widehat{H}_{0}(\tau)\right|^{-1 / 2}\right\|^{2},
$$

где $b=\left|\pi b_{1}+\xi\right|$. В силу (10) первая сумма оценивается через $C(b) \tau^{-1}\|u\|_{L_{2}(\widetilde{U} \times \Omega)}^{2}$ и удовлетворяет утверждению леммы. Оценим вторую сумму. Собственные значения оператора $-\Delta$ имеют вид $|\hat{n}|^{2}+|n|^{2}, \hat{n} \in \widetilde{\Gamma}^{\prime}, n \in \Gamma^{\prime}$. Область значений проектора $E_{\mu}$ отвечает таким парам $(\hat{n}, n)$, что $(\mu-1)^{2} \leqslant|\hat{n}|^{2}+|n|^{2}<\mu^{2}$. Отсюда вытекает, что $\left|n+\pi b_{1}+\xi\right|^{2}+|\hat{n}|^{2} \in\left[(\mu-b-1)^{2} ;(\mu+b)^{2}\right)$. Следовательно, при $\mu \geqslant[b]+2$

$$
\begin{aligned}
\left\|E_{\mu}\left|\widehat{H}_{0}(\tau)\right|^{-1 / 2}\right\|^{2} & =\max _{|\hat{n}|^{2}+|n|^{2} \in\left[(\mu-1)^{2} ; \mu^{2}\right)} \frac{1}{\left|\hat{h}_{\hat{n} n}(\tau)\right|} \\
& \leqslant \max _{\left|n+\pi b_{1}+\xi\right|^{2}+|\hat{n}|^{2} \in\left[(\mu-1-b)^{2} ;(\mu+b)^{2}\right)} \frac{\sqrt{2}}{\left|n+\pi b_{1}+\xi\right|^{2}+|\hat{n}|^{2}-\tau^{2} \mid+\tau} .
\end{aligned}
$$


Таким образом, вторая сумма в правой части формулы (13) не превосходит

$$
\begin{aligned}
C\|u\|_{L_{2}(\widetilde{U} \times \Omega)}^{2} \sum_{\mu=b+1}^{\infty} \max _{\left|n+\pi b_{1}+\xi\right|^{2}+|\hat{n}|^{2} \in\left[(\mu-1-b)^{2} ;(\mu+b)^{2}\right)} & \frac{\mu^{1-2 \delta}}{\| n+\pi b_{1}+\left.\xi\right|^{2}+|\hat{n}|^{2}-\tau^{2} \mid+\tau} \\
& \leqslant C(b, \delta) \tau^{-\delta}\|u\|_{L_{2}(\widetilde{U} \times \Omega)}^{2}
\end{aligned}
$$

по лемме 7. Но последняя величина стремится к нулю при $\tau \rightarrow+\infty$.

С точностью до обозначений следующее предложение доказано в [4, теорема $1.2]$.

Предложение 9. Пусть $V \in L_{d / 2}(\widetilde{U} \times \Omega)$. Существует такое $\tau_{0}>0$, ито при $\tau>\tau_{0}$

$$
\left\||V|^{1 / 2} u\right\|_{L_{2}(\widetilde{U} \times \Omega)} \leqslant C\|V\|_{L_{d / 2}(\widetilde{U} \times \Omega)}^{1 / 2}\left\|\left|\widehat{H}_{0}(\tau)\right|^{1 / 2} u\right\|_{L_{2}(\widetilde{U} \times \Omega)} \quad \forall u \in H_{\mathrm{per}}^{1}(\widetilde{U} \times \Omega) .
$$

\section{§4. Оценки свободного оператора}

Оператор $H\left((\pi+i \tau) b_{1}+\xi\right)$ при фиксированных $b_{1}, \xi$ будем обозначать через $H(\tau)$; мы не будем рассматривать его при других значениях квазиимпульса, поэтому это не приведет к недоразумениям. Пусть $H_{0}(\tau)$ - тот же оператор при $V=0, \sigma=0$. Заметим, что последний оператор действует на вектор-функции из $\mathbb{C}^{N}$, не перемешивая их компоненты, и является ортогональной суммой $N$ скалярных операторов, отличающихся только краевыми условиями; на каждой паре противоположных граней заданы условия Дирихле или Неймана. В связи с этим в этом параграфе везде, кроме формулировок лемм, будет предполагаться, что $N=1$.

Пусть $T: L_{2}(U \times \Omega) \rightarrow L_{2}(\widetilde{U} \times \Omega)$ - оператор, продолжающий функцию по координатам $x$ с $U$ на $\widetilde{U}$ четным образом через границы с условием Неймана и нечетным - через границы с условием Дирихле. Собственные функции оператора Лапласа в $U$ с краевыми условиями (6) переходят в собственные функции оператора Лапласа в $\widetilde{U}$ с периодическими краевыми условиями и теми же собственными значениями. Отсюда следует, что $T H_{\mathscr{C}}^{1}(U \times \Omega) \subset H_{\mathrm{per}}^{1}(\widetilde{U} \times \Omega)$. Kроме того, верны соотношения

$$
\begin{gathered}
\|T u\|_{L_{r}(\widetilde{U} \times \Omega)}=2^{k / r}\|u\|_{L_{r}(U \times \Omega)}, \quad \widehat{H}_{0}(\tau) T u=T H_{0}(\tau) u, \\
\left|\widehat{H}_{0}(\tau)\right|^{1 / 2} T u=T\left|H_{0}(\tau)\right|^{1 / 2} u
\end{gathered}
$$

а также

$$
\left|\widehat{H}_{0}(\tau)\right|^{-1 / 2} T u=T\left|H_{0}(\tau)\right|^{-1 / 2} u, \quad \tau>0,
$$

в случае, если правые часть определены. Равенства проверяются разложением по собственным функциям оператора $H_{0}(\tau)$.

Лемма 10. Для любого $1 \leqslant q<2(d-1) /(d-2)$ существует такое $\tau_{0}>0$, что при $\tau>\tau_{0}$

$\left\|\left|H_{0}(\tau)\right|^{-1 / 2} u\right\|_{L_{q}\left(\Sigma \cap(U \times \Omega) ; \mathbb{C}^{N}\right)}^{2} \leqslant C(q, \tau)\|u\|_{L_{2}\left(U \times \Omega ; \mathbb{C}^{N}\right)}^{2}, \quad C(q, \tau) \rightarrow 0, \tau \rightarrow+\infty$. 
Доказательство. Заметим, что из (15), леммы 8 и (14) при $\tau>\tau_{0}$ вытекает

$$
\begin{aligned}
\left\|\left|H_{0}(\tau)\right|^{-1 / 2} u\right\|_{L_{q}(\Sigma \cap(U \times \Omega))}^{2} & \leqslant\left\|\left|\widehat{H}_{0}(\tau)\right|^{-1 / 2} T u\right\|_{L_{q}(\widetilde{\Sigma})}^{2} \\
& \leqslant C(q, \tau)\|T u\|_{L_{2}(\widetilde{U} \times \Omega)}^{2}=2^{k} C(q, \tau)\|u\|_{L_{2}(U \times \Omega)}^{2} .
\end{aligned}
$$

Следующая лемма очевидным образом следует из (10).

Лемма 11. При $\tau \neq 0, u \in L_{2}(U \times \Omega)$

$$
\left\|\left|H_{0}(\tau)\right|^{-1 / 2} u\right\|_{L_{2}(U \times \Omega)} \leqslant(2 \pi \tau)^{-1 / 2}\|u\|_{L_{2}(U \times \Omega)} .
$$

Через $(\cdot, \cdot)$ обозначаем стандартное скалярное произведение в $L_{2}\left(U \times \Omega ; \mathbb{C}^{N}\right)$,

$$
\left(u_{1}, u_{2}\right)=\int_{U \times \Omega}\left\langle u_{1}(x, y), u_{2}(x, y)\right\rangle d x d y .
$$

Лемма 12. Пусть $u_{1}, u_{2} \in \operatorname{Dom}\left(H_{0}(\tau)\right)$. Пусть $V \in L_{d / 2}\left(U \times \Omega ; M_{N}(\mathbb{C})\right)$. Тогда

$$
\begin{aligned}
\left|\left(V u_{1}, u_{2}\right)\right| \leqslant C & \|V\|_{L_{d / 2}\left(U \times \Omega ; M_{N}(\mathbb{C})\right)} \\
& \times\left\|\left|H_{0}(\tau)\right|^{1 / 2} u_{1}\right\|_{L_{2}\left(U \times \Omega ; M_{N}(\mathbb{C})\right)}\left\|\left|H_{0}(\tau)\right|^{1 / 2} u_{2}\right\|_{L_{2}\left(U \times \Omega ; M_{N}(\mathbb{C})\right)} .
\end{aligned}
$$

Доказательство. Продолжим $V$ нулем с $U \times \Omega$ на $\widetilde{U} \times \Omega$. Имеем

$$
\left|\left(V u_{1}, u_{2}\right)\right|=\left|\left(V T u_{1}, T u_{2}\right)\right| \leqslant\left.|| V\right|^{1 / 2} T u_{1}\left\|_{L_{2}(\widetilde{U} \times \Omega)}|||V|^{1 / 2} T u_{2}\right\|_{L_{2}(\widetilde{U} \times \Omega)} .
$$

В силу предложения 9 получаем

$$
\begin{aligned}
\left|\left(V u_{1}, u_{2}\right)\right| & \leqslant C\|V\|_{L_{d / 2}(\widetilde{U} \times \Omega)}\left\|\left|\widehat{H}_{0}(\tau)\right|^{1 / 2} T u_{1}\right\|_{L_{2}(\widetilde{U} \times \Omega)}\left\|\left|\widehat{H}_{0}(\tau)\right|^{1 / 2} T u_{2}\right\|_{L_{2}(\widetilde{U} \times \Omega)} \\
& =C\|V\|_{L_{d / 2}(U \times \Omega)}\left\|T\left|H_{0}(\tau)\right|^{1 / 2} u_{1}\right\|_{L_{2}(\widetilde{U} \times \Omega)}\left\|T\left|H_{0}(\tau)\right|^{1 / 2} u_{2}\right\|_{L_{2}(\widetilde{U} \times \Omega)} \\
& =2^{k} C\|V\|_{L_{d / 2}(U \times \Omega)}\left\|\left|H_{0}(\tau)\right|^{1 / 2} u_{1}\right\|_{L_{2}(U \times \Omega)}\left\|\left|H_{0}(\tau)\right|^{1 / 2} u_{2}\right\|_{L_{2}(U \times \Omega)} .
\end{aligned}
$$

\section{§5. Доказательство теоремы 2}

Дальнейшие рассуждения проводятся по обычной схеме, см., например, [2] или [8]. Теорема 4 будет доказана, если мы установим, что для любого $v_{1} \in$ $\operatorname{Dom}(H(\tau)),\left\|v_{1}\right\|=1$, существует $v_{2}$, такое, что $\left\|v_{2}\right\|=1$ и

$$
\left|\left(H(\tau) v_{1}, v_{2}\right)-\lambda\left(v_{1}, v_{2}\right)\right| \geqslant C \tau \text { при } \tau \geqslant \tau_{0} .
$$

Рассмотрим полярное разложение

$$
H_{0}(\tau)=\Phi_{0}(\tau)\left|H_{0}(\tau)\right| .
$$

Пусть $v_{1} \in \operatorname{Dom}(H(\tau)),\left\|v_{1}\right\|=1$. Положим $v_{2}=\Phi_{0}(\tau) v_{1}$. Имеем

$$
\left(H_{0}(\tau) v_{1}, v_{2}\right)=\left\|\left|H_{0}(\tau)\right|^{1 / 2} v_{1}\right\|_{L_{2}(U \times \Omega)}^{2}=\left\|\left|H_{0}(\tau)\right|^{1 / 2} v_{2}\right\|_{L_{2}(U \times \Omega)}^{2} .
$$

Из (10) следует, что

$$
\left(H_{0}(\tau) v_{1}, v_{2}\right)=\left(\left|H_{0}(\tau)\right| v_{1}, v_{1}\right) \geqslant 2 \pi \tau .
$$

Пусть $\sigma \in L_{p}\left(\partial U \times \Omega ; M_{N}(\mathbb{C})\right), p>d-1, q=2 p^{\prime}<2(d-1) /(d-2)$. Применяя неравенство Гёльдера и лемму 10, получаем 


$$
\begin{aligned}
& \left|\int_{\Sigma \cap(U \times \Omega)}\left\langle\sigma(x, y) v_{1}(x, y), v_{2}(x, y)\right\rangle d S(x, y)\right| \\
& \leqslant\|\sigma\|_{L_{p}\left(\Sigma \cap(U \times \Omega) ; M_{N}(\mathbb{C})\right)}\left\|v_{1}\right\|_{L_{q}\left(\Sigma \cap(U \times \Omega) ; \mathbb{C}^{N}\right)}\left\|v_{2}\right\|_{L_{q}\left(\Sigma \cap(U \times \Omega) ; \mathbb{C}^{N}\right)} \\
& \leqslant C(q, \tau)\|\sigma\|_{L_{p}\left(\Sigma \cap(U \times \Omega) ; M_{N}(\mathbb{C})\right)} \\
& \quad \times\left\|\left|H_{0}(\tau)\right|^{1 / 2} v_{1}\right\|_{L_{2}\left(U \times \Omega ; \mathbb{C}^{N}\right)}\left\|\left.H_{0}(\tau)\right|^{1 / 2} v_{2}\right\|_{L_{2}\left(U \times \Omega ; \mathbb{C}^{N}\right)} .
\end{aligned}
$$

Пусть

$$
V=V_{\varepsilon}+V_{\infty}, \quad\left\|V_{\varepsilon}\right\|_{L_{d / 2}\left(U \times \Omega ; M_{n}(\mathbb{C})\right)} \leqslant \varepsilon, V_{\infty} \in L_{\infty}\left(U \times \Omega ; M_{N}(\mathbb{C})\right) .
$$

В силу лемм 11 и 12

$$
\begin{aligned}
& \left|\int_{U \times \Omega}\left\langle V(x, y) v_{1}(x, y), v_{2}(x, y)\right\rangle d x d y\right| \\
& \quad \leqslant C\left(\varepsilon+C(\varepsilon) \tau^{-1}\right)\left\|\left|H_{0}(\tau)\right|^{1 / 2} v_{1}\right\|_{L_{2}\left(U \times \Omega ; \mathbb{C}^{N}\right)}\left\|\left.H_{0}(\tau)\right|^{1 / 2} v_{2}\right\|_{L_{2}\left(U \times \Omega ; \mathbb{C}^{N}\right)} .
\end{aligned}
$$

Объединяя оценки, получаем

$$
\begin{aligned}
\left|\left(H(\tau) v_{1}, v_{2}\right)-\lambda\left(v_{1}, v_{2}\right)\right| & \\
\geqslant & \left(H_{0}(\tau) v_{1}, v_{2}\right)-|\lambda|-\left|\int_{\Sigma \cap(U \times \Omega)}\left\langle\sigma(x, y) v_{1}(x, y), v_{2}(x, y)\right\rangle d S(x, y)\right| \\
& \quad-\left|\int_{U \times \Omega}\left\langle V(x, y) v_{1}(x, y), v_{2}(x, y)\right\rangle d x d y\right| \\
\geqslant & \left(H_{0}(\tau) v_{1}, v_{2}\right)-|\lambda|-C\left(\varepsilon+C(q, \tau)\|\sigma\|_{L_{p}(\Sigma \cap(U \times \Omega))}\right. \\
& \left.\quad+C(\varepsilon) \tau^{-1}\right)||\left|H_{0}(\tau)\right|^{1 / 2} v_{1}\left\|_{L_{2}(U \times \Omega)}\right\|\left|H_{0}(\tau)\right|^{1 / 2} v_{2} \|_{L_{2}(U \times \Omega)} \\
= & \left(H_{0}(\tau) v_{1}, v_{2}\right)-|\lambda|-C\left(\varepsilon+\widetilde{C}(q, \tau)+C(\varepsilon) \tau^{-1}\right)\left(H_{0}(\tau) v_{1}, v_{2}\right) \\
\geqslant & 2 \pi \tau\left(1-C\left(\varepsilon+\widetilde{C}(q, \tau)+C(\varepsilon) \tau^{-1}\right)\right)-|\lambda|,
\end{aligned}
$$

где $\widetilde{C}(q, \tau)=C(q, \tau)\|\sigma\|_{L_{p}(\Sigma \cap(U \times \Omega))}$. Последнее неравенство завершает доказательство, поскольку $C, C(\varepsilon)$ не зависят от $\tau, C$ не зависит от $\varepsilon, \widetilde{C}(q, \tau) \rightarrow 0$, а $\lambda$ фиксировано.

Замечание 13. Утверждение теоремы 3 остается верным, если в качестве $U$ взять $\left[0 ; a_{1}\right] \times \cdots \times\left[0 ; a_{k}\right] \times \mathbb{T}^{l}$, где $\mathbb{T}^{l}=\mathbb{R}^{l} / \Gamma_{l}, \Gamma_{l} \subset \mathbb{R}^{l}-$ некоторая $l$-мерная решетка, $k+l+m=d$. Это соответствует добавлению к $U$ граней с периодическими краевыми условиями. С точностью до обозначений, доказательство дословно повторяет доказательство теоремы 3.

Замечание 14. В скалярном случае (т. е. при $N=1$ ) в качестве $U$ вместо параллелепипеда можно рассматривать гладкое компактное риманово многообразие без края. Требования к $\Sigma$ и $\sigma$ будут теми же, но условие на электрический потенциал будет иметь вид $V \in L_{p, l o c}\left(U \times \mathbb{R}^{m}\right), p>d / 2$, так как вместо результатов работы [4] придется применять результаты из [8].

\section{ЛитЕРАТУРА}

[1] М. Ш. Бирман, Т. А. Суслина, Р. Г. Штеренберг, Абсолютная непрерьвность двумерного оператора Шрёдингера с дельта-потенциалом, сосредоточенным на периодической системе кривых, Алгебра и анализ, 12:6 (2000), 140-177. 
[2] М. Ш. Бирман, Т. А. Суслина, Периодический магнитный гамильтониан с переменной метрикой. Проблема абсолютной непрерьвности, Алгебра и анализ, 11:2 (1999), 1-40.

[3] N. Burq, P. Gérard, N. Tzvetkov, Restrictions of the Laplace-Beltrami eigenfunctions to submanifolds, Duke Math. J., 138:3 (2007), 445-486.

[4] L. Danilov, On absolute continuity of the spectrum of a periodic magnetic Schrödinger operator, J. Phys. A: Math. Theor., 42:27 (2009), 275204.

[5] Т. Като, Теория возмущений линейных операторов, Мир, М., 1972.

[6] И. В. Качковский, Теорема Стейна-Томаса для тора и периодический оператор Шрёдингера с сингулярным потенииалом, Алгебра и анализ, 24:6 (2012), 124-138.

[7] И. В. Качковский, Н. Д. Филонов, Абсолютная непрерывность спектра периодического оператора Шрёдингера в многомерном иилиндре, Алгебра и анализ, 21:1 (2009), 133-152.

[8] И. Качковский, Н. Филонов, Абсолютная непрерывность спектра периодического оператора Шрёдингера в слое и в гладком иилиндре, Краевые задачи математической физики и смежные вопросы теории функций, 41, Зап. научн. сем. ПОМИ, 385 (2010), 69-82.

[9] P. Kuchment, Floquet Theory for Partial Differential Equations, Birkhauser, Basel, 1993.

[10] P. Kuchment, S. Levendorskii, On the structure of spectra of periodic elliptic operators, Trans. Amer. Math. Soc., 354:2 (2001), 537-569.

[11] А. Прохоров, Н. Филонов, О спектре периодического оператора Максвелла в иилиндре (в печати).

[12] М. Рид, Б. Саймон, Методъ современной математической физики, m. 4, Анализ операторов, Мир, М., 1982.

[13] A. Sobolev, J. Walthoe, Absolute continuity in periodic waveguides, Proc. London Math. Soc., 85:3 (2002), 717-741.

[14] E. Shargorodsky, A. V. Sobolev, Quasiconformal mappings and periodic spectral problems in dimension two, J. Anal. Math., 91 (2003), 67-103.

[15] T. A. Suslina, On the absence of eigenvalues of a periodic matrix Schrödinger operator in a layer, Russ. J. Math. Phys., 8:4 (2001), 463-486.

[16] Т. А. Суслина, Абсолютная непрерьвность спектра периодического оператора Максвелла в слое, Краевые задачи математической физики и смежные вопросы теории функций, 32, Зап. научн. сем. ПОМИ, 288 (2002), 232-255.

[17] Т. А. Суслина, Р. Г. Штеренберг, Абсолютная непрерывность спектра оператора Шрёдингера с потенциалом, сосредоточенным на периодической системе гиперповерхностей, Алгебра и анализ, 13:5 (2001), 197-240.

[18] Т. А. Суслина, Р. Г. Штеренберг, Абсолютная непрерывность спектра магнитного оператора Шрёдингера с метрикой в двумерном периодическом волноводе, Алгебра и анализ, 14:2 (2002), 159-206.

[19] L. Thomas, Time dependent approach to scattering from impurities in a crystal, Comm. Math. Phys., 33 (1973), 335-343.

[20] Z. Shen, On absolute continuity of the periodic Schrödinger operators, Intern. Math. Res. Notes, 2001:1 (2001), 1-31.

[21] Р. Г. Штеренберг, Абсолютная непрерьвность спектра двумерного периодического оператора Шрёдингера с сильно подчиненным магнитным потенииалом, Исследования по линейным операторам и теории функций, 31, Зап. научн. сем. ПОМИ, 303 (2003), 279-320. 